\title{
Characterization of best linear unbiased estimates generated from national genetic evaluations of reproductive performance, survival, and milk yield in dairy cows
}

\author{
F. L. Dunne, ${ }^{*} \dagger$ M. M. Kelleher, $\ddagger$ S. W. Walsh, $†$ and D. P. Berry ${ }^{* 1}$ \\ *Teagasc, Animal and Grassland Research and Innovation Center, Moorepark, Fermoy, Co. Cork, Ireland P61 C996 \\ tWaterford Institute of Technology, Cork Road, Waterford, Co. Waterford, Ireland X91 K0EK \\ flrish Cattle Breeding Federation, Bandon, Co. Cork, Ireland P72 X050
}

\begin{abstract}
Genetic evaluations decompose an observed phenotype into its genetic and nongenetic components; the former are termed BLUP with the solutions for the systematic environmental effects in the statistical model termed best linear unbiased estimates (BLUE). Geneticists predominantly focus on the BLUP and rarely consider the BLUE. The objective of this study, however, was to define and quantify the association between 8 herd-level characteristics and BLUE for 6 traits in dairy herds, namely (1) age at first calving, (2) calving to first service interval (CFS), (3) number of services, (4) calving interval (CIV), (5) survival, and (6) milk yield. Phenotypic data along with the fixed and random effects solutions were generated from the Irish national multi-breed dairy cow fertility genetic evaluations on 3,445,557 cows; BLUE for individual contemporary groups were collapsed into mean herd-year estimates. Data from 5,707 spring-calving herds between the years 2007 and 2016 inclusive were retained; association analyses were undertaken using linear mixed multiple regression models. Pearson coefficient correlations were used to quantify the relationships among individual trait herd-year BLUE, and transition matrices were used to understand the dynamics of mean herd BLUE estimates over years. Based on the mean annual trends in raw, BLUP, and BLUE, it was estimated that BLUE were associated with at least two-thirds of the improvement in CIV and milk production over the past $10 \mathrm{yr}$. Milk recording herds calved heifers for the first time on average $15 \mathrm{~d}$ younger, had an almost $2 \mathrm{~d}$ longer CFS but $2.3 \mathrm{~d}$ shorter CIV than non-milk-recording herds. Larger herd sizes were associated with worse BLUE for both CFS and CIV. Expanding herds and herds that had the highest proportion of cows born on
\end{abstract}

Received February 1, 2018.

Accepted April 12, 2018.

${ }^{1}$ Corresponding author: donagh.berry@teagasc.ie the farm itself, on average, calved heifers younger and had shorter CIV. By separating the raw performance of a selection of herds into their respective BLUE and BLUP, it was possible to identify herds with inferior management practices that were being compensated by superior genetics; similarly, herds were identified with superior BLUE, but because of their inferior genetic merit, were not reaching their full potential. This suggests that BLUE could have a pivotal role in a tailored decision support tool that would enable producers to focus on the most limiting factor hindering them from achieving their maximum performance.

Key words: best linear unbiased estimate, dairy, fertility, management

\section{INTRODUCTION}

Animal breeders have traditionally focused on improving the mean genetic merit of a population in the pursuit of enhanced performance. Animal performance, however, is a function of both the genotype of the animal and the environment it is (and was) exposed to. Failure to improve animal management concomitant with the requirements of the genetically elite germplasm hampers the actual realization of the benefit in genetic gain. Genetic evaluations apportion the phenotype of an individual into its estimated additive genetic merit (i.e., BLUP) and the contributing environmental or management influences (i.e., best linear unbiased estimates; BLUE). Whereas the BLUP have been extensively used in animal breeding programs (Meuwissen et al., 2001; Muir, 2007; Patry and Ducrocq, 2011), BLUE have received considerably less attention.

Most genetic evaluations consider as fixed effects some measure of temporal herd groupings such as the contemporary group of herd-year-season (van Bebber et al., 1997; Calus et al., 2005) or just herd-year (Englishby et al., 2017). Bastin et al. (2009) proposed using such herd-year BLUE to provide dairy producers with retrospective advice on feeding and management 
strategies. Bastin et al. (2009) found that by analyzing the BLUE associated with the milk urea concentration of milk samples, an association could be made between dietary $\mathrm{CP}$ and the dietary management of the animals. This information could then be used to provide farmers with ongoing feed management advice (Bastin et al., 2009). Similarly, Caccamo et al. (2008) included a herd-test-day effect in the statistical analyses of dairy cow milk performance data to account for short-term management changes such as changes in feed ration. Englishby et al. (2017) used BLUE to independently quantify the association between management environments and the variability in beef carcass characteristics after accounting for differences in additive genetic merit; Englishby et al. (2017) achieved this by including the random effects of both animal genetic merit and the contemporary group of finishing herd-year in the statistical model. Studies are however lacking, in dairy cattle at least, on the macro-environmental and management characteristics describing the BLUE.

The objective of the present study was to describe herd-level related factors contributing to herd-year BLUE and to understand the inter-relationships among herd-year BLUE for fertility, milk, and survival in dairy cows. The information generated has the potential to be used within a management decision support tool to enable producers to make more informed value-creating decisions.

\section{MATERIALS AND METHODS}

All data used in the present study originated from the Irish Cattle Breeding Federation database, Bandon, Co. Cork, Ireland.

\section{Genetic Evaluation}

The edited phenotypes and associated fixed effects used in the Irish national multi-breed dairy cow fertility genetic evaluations were available; data on a total of $3,445,557$ cows were available, which consisted of predominantly Holstein-Friesian (91\%) with the remainder being mainly crossbred animals. A national genetic evaluation was subsequently run in Mix99 (MiX99 Development Team, 2015) and the fixed effects (and random effects) solutions generated. The national dairy cow fertility genetic evaluation used in Ireland is a 23 $\times 23$ multi-trait evaluation that includes age at first calving, calving to first service interval from parity 1 to 3 as separate traits, number of services from parity 1 to 3 as separate traits, calving interval from parity 1 to 5 as separate traits, 305-d milk yield from parity 1 to 5 as separate traits, survival from parity 1 to 5 as separate traits, and lifespan; lifespan was not considered further.
Details of the editing procedures, statistical models, and variance components for each trait are described in Berry et al. (2013). Fixed effects common to all traits were the contemporary group of herd-year-season of calving (or birth when the dependent variable was age at first calving), age at calving (except when the dependent variable was age at first calving), heterosis, and recombination loss coefficients. Contemporary groups are created for each trait using the procedure outlined in Berry et al. (2013) based on algorithms proposed by Schmitz et al. (1991) and Crump et al. (1997).

\section{Definition of Herd-Year BLUE Effects}

Contemporary group BLUE from the national genetic evaluations were available. Contemporary group effect estimates were not necessarily available for all traits; for example, herds that do not participate in milk recording would not have a contemporary group effect estimate for 305-d milk yield but could have a contemporary group effect estimate for any of the fertility traits. A given herd-year could have several contemporary groups because clusters of animals may calve at different periods of the year within a herd and therefore would be in different contemporary groups; therefore, the average contemporary group effect estimate within each herd-year, weighted by the respective number of records in each contemporary group, was calculated. Only data from herds with information for each year in the 10-yr period from 2007 to 2016 inclusive were retained; annual herd-year BLUE for each of the $10 \mathrm{yr}$ were available from 8,873 herds (i.e., 88,730 herd-years).

\section{Herd-Year Level Characteristics}

Herd-year characteristics of interest included: (1) geographical location, (2) whether or not the herd was milk recording, (3) herd size, (4) herd expansion rate, (5) herd-level use of AI, (6) the proportion of cows in the herd that were born in that herd (i.e., not purchased), (7) the proportion of cows in the herd that were registered with a breed society, and (8) the proportion of cows in the herd that calved in the first $42 \mathrm{~d}$ of the calving season.

Geographical location was available for all herds in the Republic of Ireland on a county basis; herds originated from a total of 26 counties with the 3 largest counties in the country further subdivided into north and south. A herd was defined as a milk recording herd in a given year based on the presence of milk recording data for that herd in the national database for that year; all data from milk-recorded herds are stored in the central database. 
Herd size for a given herd-year was based on the number of calving events in that year. The trend in annual herd size was used to calculate the rate of expansion using an approach similar to that described in detail by Jago and Berry (2011); a linear robust regression was used to calculate the expansion rate of each herd-year from 2007 to 2016 inclusive. To calculate the rate of expansion for a given year, the herd size of that year as well as the 2 flanking years were used in the robust regression; the exception were the years 2007 and 2008 as well as 2015 and 2016, but in these cases, 5 yr of data were always included. The resulting linear coefficient was used to categorize the rate of herd expansion as outlined in Jago and Berry (2011); herds deemed not to be expanding were those with a negative linear regression coefficient or a linear regression coefficient not different (i.e., $P>0.05$ ) from zero.

The extent of AI usage in a given herd-year was calculated as the proportion of cows that calved in that herd-year that had received at least one $\mathrm{AI}$ in that year. In Ireland it is a legal requirement to register all bovine birth events including the herd of birth; the proportion of cows calving in a herd in a given year that were actually born in that herd was therefore available. Similarly, the proportion of cows calving in a given herd-year that were registered with a breed society was also generated.

Irish dairy herds are typically seasonal calving with the majority of cows calving in a short number of months in the spring (Berry et al., 2013); the start of the spring calving season in a given herd was characterized by a minimum of 5 cows calving within a 14-d period (Berry et al., 2013). Similar to Berry et al. (2013), the proportion of calving events within the first $42 \mathrm{~d}$ of the calving season relative to the number of cows that calved that year was calculated per herd-year.

\section{Data Editing and Categorization of Herd Characteristic Variables}

Only herds that had $\geq 30$ calving events in all $10 \mathrm{yr}$ (5,707 herds) were retained. Furthermore, only springcalving herds, which predominate in Ireland (Berry et al., 2013), were retained; in 2016, $86 \%$ of calves born to a dairy sire were born during the months of January to April, inclusive (DAFM, 2016). A spring-calving herd was defined as a herd where at least $80 \%$ of calving events occurred between January and June, inclusive. For the analysis of herd characteristics, a single herdyear BLUE for each of the 5 traits that contained multiple parities (i.e., calving to first service interval, calving interval, number of services, milk yield, and survival) was calculated as the weighted (by number of records per parity) average across all 3 (i.e., calving to first service interval and number of services) or 5 (i.e., calving interval, milk yield, and survival) parities. Only average herd-year BLUE for calving interval, milk yield, and survival were retained if a herd-year BLUE was available for at least 4 of the parities each containing a minimum of 5 records; average BLUE for calving to first service interval and number of services required herd-year BLUE to be available for at least 2 parities with a minimum of 5 records per parity. The mean herd-year BLUE of each collapsed trait was subsequently recoded to be relative to the mean of the entire population.

Each of the herd-level characteristics, with the exception of geographical location and milk recording, were stratified into 5 categories; as contemporary group effects were not available for all traits, to accurately represent the population distribution for herd size and the proportion of AI used on farm, the threshold values categorizing each characteristic varied per BLUE (Supplemental Table S1; https://doi.org/10.3168/jds .2018-14529). Transition matrices were generated to investigate the dynamics of herd BLUE between adjacent years. Herd-year BLUE for each trait were stratified separately into 5 strata of equal size based on the annual herd performance for each individual trait and subsequently averaged across years; the first stratum represented the top-performing herd-year BLUE for each trait and the fifth stratum represented the worstperforming herd-year BLUE. For example, the highest milk yielding herd-year BLUE were represented in stratum 1, whereas stratum 5 represented the lowest milk yielding herd-year BLUE; similarly the shortest BLUE for calving interval was represented in stratum 1 and the longest BLUE for calving interval was in stratum 5. Transition matrices between adjacent years were generated and averaged.

\section{Statistical Analyses}

Pearson coefficient correlations were used to estimate the inter- and intra-relationships between parityspecific BLUE for all traits. Correlations among all traits at an individual parity level were estimated for each year separately, and subsequently averaged across years; similarly, correlations between the single herdyear BLUE were estimated for each year separately and then averaged across years.

The association between each herd-level characteristic and each of the 6 averaged BLUE (i.e., age at first calving, calving to first service interval, calving interval, number of services, survival, and milk yield) was quantified using a linear mixed multiple regression model in SAS 9.4 software (SAS Institute Inc., Cary, $\mathrm{NC}$ ), where the dependent variable was the herd-year average BLUE. The fixed effects in the model were 
Table 1. Within-parity correlations (SE in parentheses) between annual herd-year best linear unbiased estimates for calving to first service interval (CFS), number of services (NSV), calving interval (CIV), survival (SU) and milk, averaged across the years 2007 to 2016 , with the exception of age at first calving (AFC), which was averaged across the years 2007 to 2014

\begin{tabular}{|c|c|c|c|c|c|c|}
\hline $\begin{array}{l}\text { Main } \\
\text { trait }\end{array}$ & Subtrait & \multicolumn{5}{|c|}{ Parity } \\
\hline \multirow[t]{4}{*}{$\overline{\mathrm{AFC}}$} & CFS & $-0.07^{* * *}(0.007)$ & $-0.03^{* * *}(0.007)$ & $0.00(0.007)$ & & \\
\hline & CIV & $0.10^{* * *}(0.005)$ & $0.09^{* * *}(0.005)$ & $0.07^{* * *}(0.006)$ & $0.09 * * *(0.006)$ & $0.03^{* * *}(0.007)$ \\
\hline & $\mathrm{SU}$ & $0.02^{* * *}(0.005)$ & $0.01(0.005)$ & $0.02^{* * *}(0.006)$ & $-0.03^{* * *}(0.006)$ & $0.02 * *(0.006)$ \\
\hline & Milk & $-0.10^{* * *}(0.007)$ & $-0.14^{* * *}(0.007)$ & $-0.13^{* * *}(0.007)$ & $-0.14^{* * *}(0.007)$ & $-0.14 * * *(0.007)$ \\
\hline CFS & Milk & $0.07^{* * *}(0.007)$ & $0.05^{* * *}(0.007)$ & $0.07^{* * *}(0.007)$ & & \\
\hline \multirow[t]{3}{*}{ NSV } & CIV & $0.34^{* * *}(0.006)$ & $0.34^{* * *}(0.006)$ & $0.31^{* * *}(0.006)$ & & \\
\hline & SU & $-0.16^{* * *}(0.006)$ & $-0.14^{* * *}(0.006)$ & $-0.14 * * *(0.006)$ & & \\
\hline & Milk & $-0.01(0.007)$ & $0.01(0.007)$ & $0.02^{* * *}(0.007)$ & & \\
\hline \multirow[t]{2}{*}{ CIV } & $\mathrm{SU}$ & $0.03^{* * *}(0.005)$ & $0.01(0.005)$ & $0.05^{* * *}(0.005)$ & $0.04 * * *(0.005)$ & $0.16^{* * *}(0.006)$ \\
\hline & Milk & $0.00(0.006)$ & $0.01 *(0.006)$ & $0.05 * * *(0.006)$ & $0.05 * * *(0.006)$ & $0.06^{* * *}(0.007)$ \\
\hline
\end{tabular}

those describing the herd characteristics; year was forced into all models. Herd was included as a repeated effect with the appropriate covariance structures assumed among repeated records; the most appropriate covariance structure was based on the Akaike's information criterion (Bozdogan, 2000). The repeatability for each BLUE was also estimated from the mixed model. The transition matrices were used to quantify the probability of a herd remaining or changing BLUE category between adjacent years for each of the 6 traits investigated.

\section{RESULTS}

\section{Correlations}

Calving to first service interval BLUE between parities 1 to 3 were weakly correlated with each other (0.31 to $0.39 ; \mathrm{SE}=0.006 ; P<0.001$ ). The correlation between number of services BLUE across the first 3 parities ranged from 0.46 (between parity 1 and 3 ) to
0.49 (between parity 1 and 2$)(\mathrm{SE}=0.006 ; P<0.001)$. Calving interval BLUE between the first 5 parities were weakly correlated ( 0.13 to $0.26 ; \mathrm{SE}=0.005$ to $0.006 ; P$ $<0.001)$ with each other. Survival BLUE were weakly correlated ( 0.11 to $0.21 ; \mathrm{SE}=0.005$ to $0.006 ; P<0.001$ ) among parities 1 to 5 . Strong correlations existed between milk yield BLUE in each of the 5 parities varying from 0.81 (between parity 1 and 5) to 0.90 (between parity 2 and $3 ; \mathrm{SE}=0.006$ to $0.007 ; P<0.001$ ). Weak correlations existed between BLUE of different traits, within the same parity, ranging from -0.17 (between calving to first service interval and number of services in parity 2 ; $\mathrm{SE}=0.006 ; P<0.001$ ) to 0.34 (between number of services and calving interval, in parity 1 ; SE $=0.006 ; P<0.001 ;$ Table 1$)$. The single parity BLUE that were collapsed into individual mean BLUE were weakly correlated with each other ranging from -0.18 (between calving to first service interval and number of services; $\mathrm{SE}=0.006 ; P<0.001$ ) to 0.31 (between number of services and calving interval; $\mathrm{SE}=0.008 ; P$ $<0.001 ;$ Table 2).

Table 2. Average correlations (SE in parentheses) across years (above diagonal) as well as the minimum and maximum correlations for any given year (below diagonal) between individual herd-year best linear unbiased estimates

\begin{tabular}{lcccrrr}
\hline Trait $^{1}$ & AFC & CFS & NSV & CIV & SU \\
\hline AFC & & $-0.05^{* * *}(0.007)$ & $0.01(0.007)$ & $0.12^{* * *}(0.007)$ & $-0.01(0.007)$ & Milk \\
CFS & $-0.09,-0.01$ & & $-0.18^{* * *}(0.006)$ & $0.16^{* * *}(0.008)$ & $-0.05^{* * *}(0.007)$ & $0.12^{* * *}(0.008)$ \\
NSV & $-0.02,0.03$ & $-0.22,-0.15$ & & $0.31^{* * *}(0.008)$ & $-0.13^{* * *}(0.007)$ & $-0.01(0.008)$ \\
CIV & $0.08,0.15$ & $0.13,0.23$ & $0.25,0.35$ & & $0.02^{* * *}(0.007)$ & $0.00(0.007)$ \\
SU & $-0.06,0.06$ & $-0.09,-0.01$ & $-0.16,-0.10$ & $-0.03,0.08$ & $0.03^{* * *}(0.007)$ \\
Milk & $-0.14,-0.07$ & $-0.02,0.09$ & $-0.04,0.04$ & $-0.07,0.06$ & $-0.03,0.10$ & \\
\hline
\end{tabular}

$\overline{{ }^{1} \mathrm{AFC}}=$ age at first calving; $\mathrm{CFS}=$ calving to first service interval; $\mathrm{NSV}=$ number of services; $\mathrm{CIV}=$ calving interval; $\mathrm{SU}=$ survival; milk $=$ milk yield.

$* * * P<0.001$. 


\section{Annual Best Linear Unbiased Estimate Trends}

Mean annual BLUE for age at first calving spiked in length twice (i.e., in 2008 and 2012) to approximately $13 \mathrm{~d}$ on both occasions and then fell to $-6.7 \mathrm{~d}$ in 2014 (Figure 1). The mean annual BLUE for calving to first service interval lengthened between the years 2007 to 2009 and peaked in the year 2013 (2.9 d); after a sharp fall to $-1.1 \mathrm{~d}$ in 2014, the mean annual BLUE for calving to first service interval began to lengthen again (Figure 1). Mean annual BLUE for number of services reduced between the years 2007 and 2015, resulting in a difference of 0.13 services (from 0.06 to -0.07 serves); during the same period of time, calving interval BLUE shortened by $6.9 \mathrm{~d}$ (Figure 1). Mean annual BLUE for survival reduced from 2007 to 2013 (Figure 1), after which it began to improve. After a reduction in mean annual BLUE for milk yield from $126 \mathrm{~kg}$ in 2007 to $-391 \mathrm{~kg}$ in 2009, mean annual BLUE for milk yield increased to $207 \mathrm{~kg}$ in 2015 (Figure 1).

\section{Repeatability and Transition Matrices}

The repeatability of herd-year BLUE for both age at first calving (0.49) and number of services (0.35) across years was moderate; the mean BLUE for calving to first service interval, calving interval, and survival across years was, however, lowly repeatable, ranging from 0.20 to 0.28. The mean BLUE for milk yield across years was highly repeatable at 0.74 .

Based on the transition matrices between consecutive years (Table 3; SE ranged from 0.11 to 2.08), herds generally had a greater probability of remaining within the same stratum from one year to the next. The probability of transitioning to an alternative stratum reduced as the distance between strata compared increased. For example, herds in the stratum representing the highest yielding mean BLUE for milk had a $74 \%$ probability of remaining in that stratum the following year but only a $0.42 \%$ probability of transitioning to the lowest yielding stratum; herds in the lowest milk yielding stratum
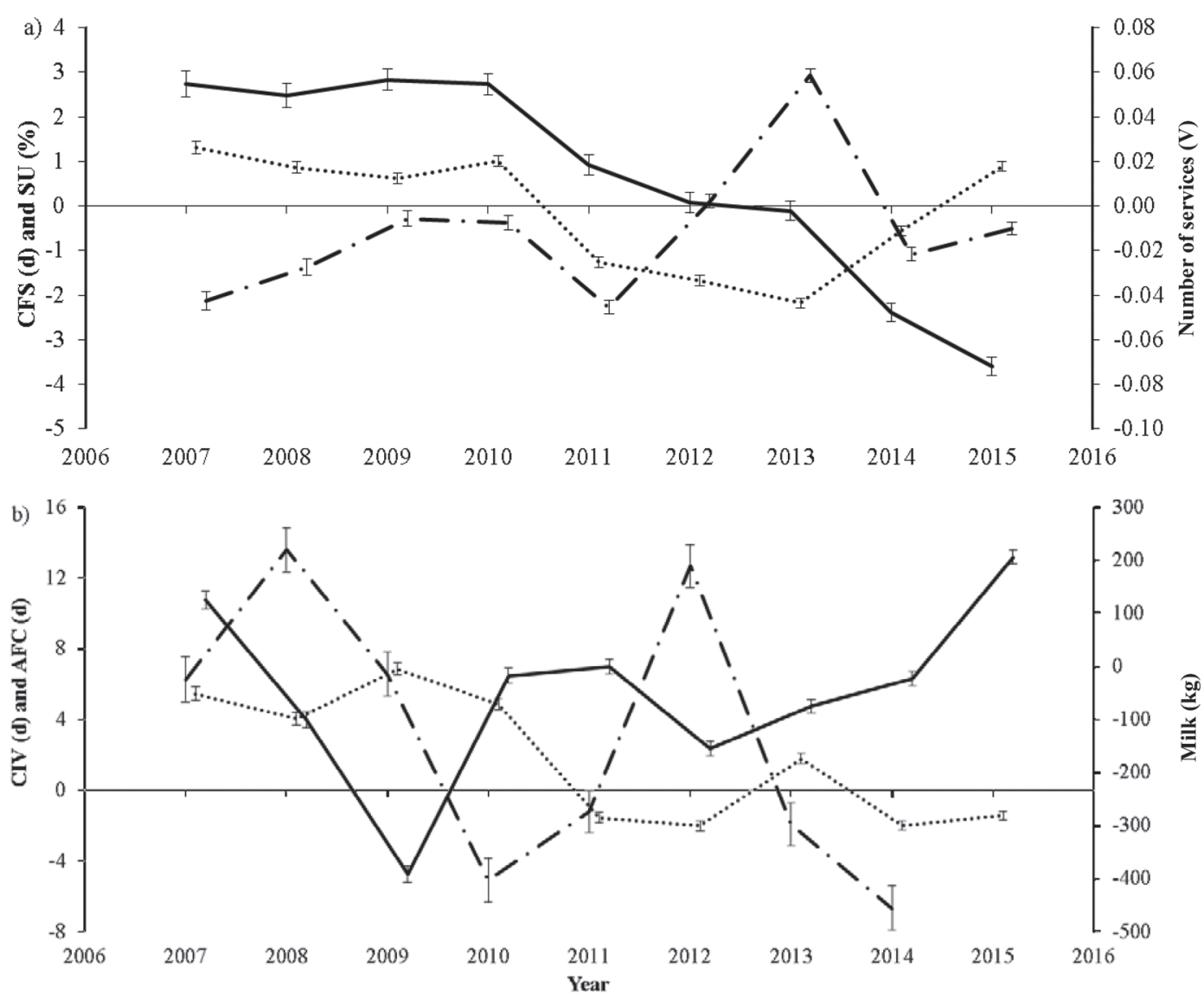

Figure 1. Annual LSM (1 SE each side represented as error bars) of the best linear unbiased estimates for (a) survival (SU; dotted line), calving to first service (CFS; dashed-dotted line), and number of services (solid line), and (b) calving interval (CIV; dotted line), age at first calving (AFC; dashed-dotted line), and milk yield (milk; solid line). 
Table 3. Transition matrices between consecutive years for herd-level best linear unbiased estimate strata for each trait (SE in parentheses)

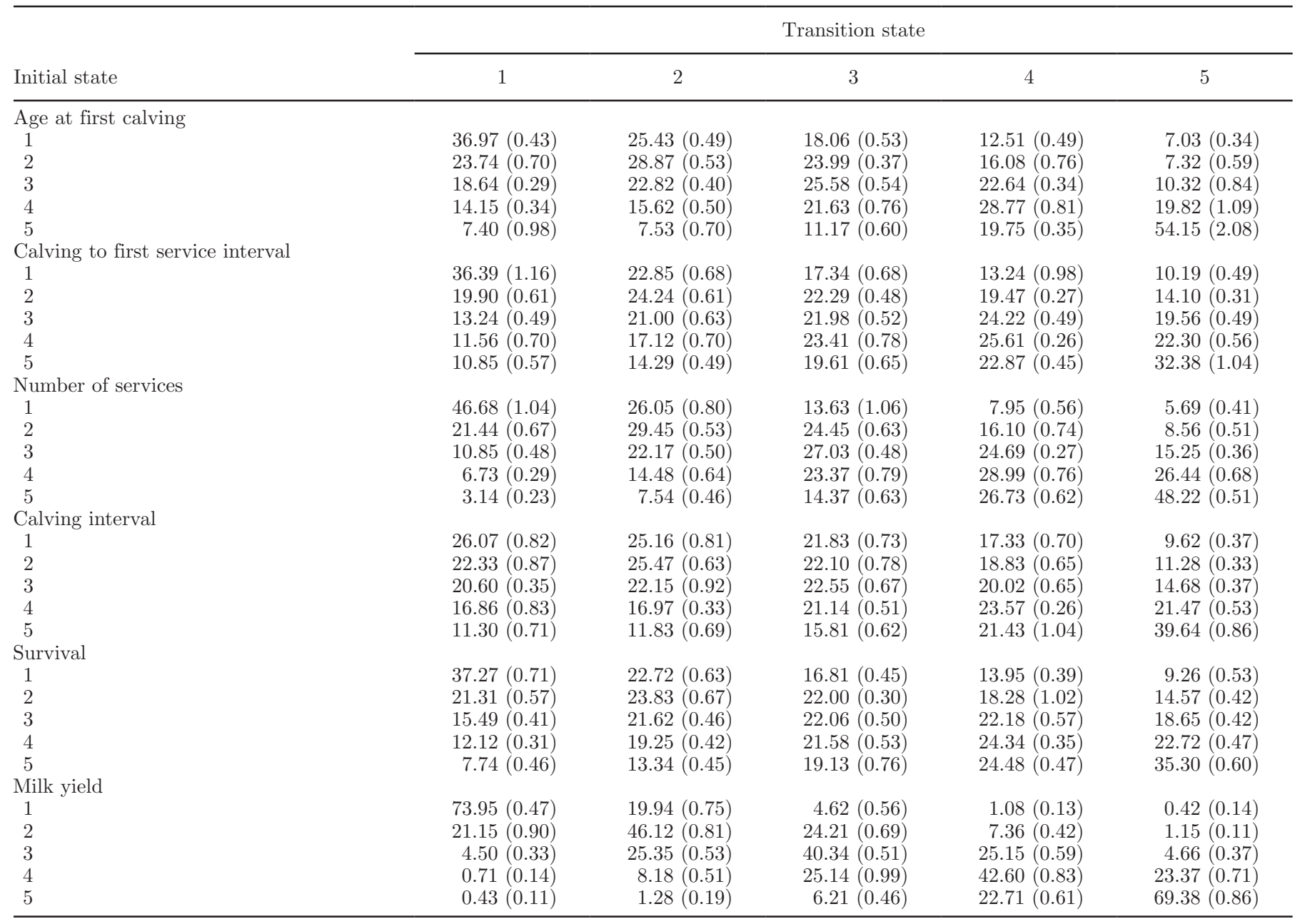

had a $69 \%$ probability of remaining in that stratum the following year and a $0.43 \%$ probability of transitioning to the highest yielding stratum the subsequent year (Supplemental Figure S1; https://doi.org/10.3168/jds .2018-14529). A sensitivity analysis was conducted by increasing the number of strata used to characterize the herd-year BLUE. However, the same phenomenon reoccurred whereby the probability of transitioning into an alternative stratum decreased as the distance between the strata increased.

\section{Herd Level Characteristics}

Least squares means for age at first calving BLUE varied from -12.5 to $46 \mathrm{~d}$ depending on geographical location, whereas mean BLUE for calving to first service interval differed across geographical location by 5.2 d. Least squares means for number of services BLUE ranged from -0.06 to 0.13 depending on geographical location. A difference of $12.8 \mathrm{~d}$ for mean calving interval BLUE, 7.3\% for mean BLUE for survival, and 2,280 $\mathrm{kg}$ for mean BLUE for milk yield existed when comparing the respective extreme geographical locations. Milk-recording herds calved heifers for the first time on average $15 \mathrm{~d}$ younger, had a calving to first service interval that was almost $2 \mathrm{~d}$ longer, and had a calving interval that was $2.3 \mathrm{~d}$ shorter compared with herds that did not milk record; however, a minimal difference in the number of services (0.01 serves) and survival $(0.4 \%)$ existed depending on whether or not the herd was milk recorded.

A younger mean BLUE for age at first calving was associated with larger herds and herds that expanded at a faster rate. A similar trend was found in herds that used a greater proportion of AI and had a greater proportion of calves born on the farm. Herds that had a greater proportion of cows calving in the first $42 \mathrm{~d}$ of the calving season as well as an increased proportion 
of cows that were registered with a breed society had a tendency to also be associated with a younger mean BLUE for age at first calving (Figure 2).

A longer mean BLUE for calving to first service interval was associated with larger herd size and a faster expansion rate, as well as being associated with a greater proportion of animals born on the farm, a greater proportion of cows registered with a breed society, and a greater proportion of cows that calved in the first 42 $\mathrm{d}$ of the calving season. A reduction in the proportion of AI used on farm was associated with a longer mean BLUE for calving to first service interval (Figure 3).

Very little variability existed in the mean BLUE for number of services across different herd sizes, expansion rates, and the proportion of animals born on the farm. There was a tendency for the mean BLUE for number of services to increase in association with increases in the proportion of AI used on farm and the proportion of animals registered with a breed society as well as a decrease in the proportion of cows that calved within the first $42 \mathrm{~d}$ of the calving season (Figure 4).

Longer mean BLUE for calving interval was associated with larger herds, herds that were not expanding, herds that purchased a greater proportion of dairy cows, and herds that had a greater proportion of animals registered with a breed society; a reduction in the proportion of cows that calved within the first $42 \mathrm{~d}$ of the calving season as well as the proportion of AI used were both associated with a longer mean BLUE for calving interval (Figure 5).

Inferior mean BLUE for survival was associated with an increase in herd size, a static expansion rate, and an increase in the number of animals purchased. An increase in the proportion of animals registered with a breed society and a decrease in the proportion of AI used were both associated with poorer mean BLUE for survival. The proportion of cows that calved within the first $42 \mathrm{~d}$ of the calving season had no obvious association with mean BLUE for survival (Figure 6).

A tendency was observed for mean BLUE for milk yield to increase in association with both a reduction in herd size and a slower rate of expansion; an increase in mean BLUE for milk yield was associated with a greater proportion of cows born on farm, a greater proportion of AI used, as well as a greater proportion of cows that calved within the first $42 \mathrm{~d}$ of the calving season. A large increase in mean BLUE for milk yield was associated with an increased proportion of animals registered with a breed society (Figure 7).

\section{DISCUSSION}

The motivation for the present study was to quantify the inter-herd variability in performance in a given year after accounting for differences in genetic merit but also elucidate the factors associated with the herd-year phe-

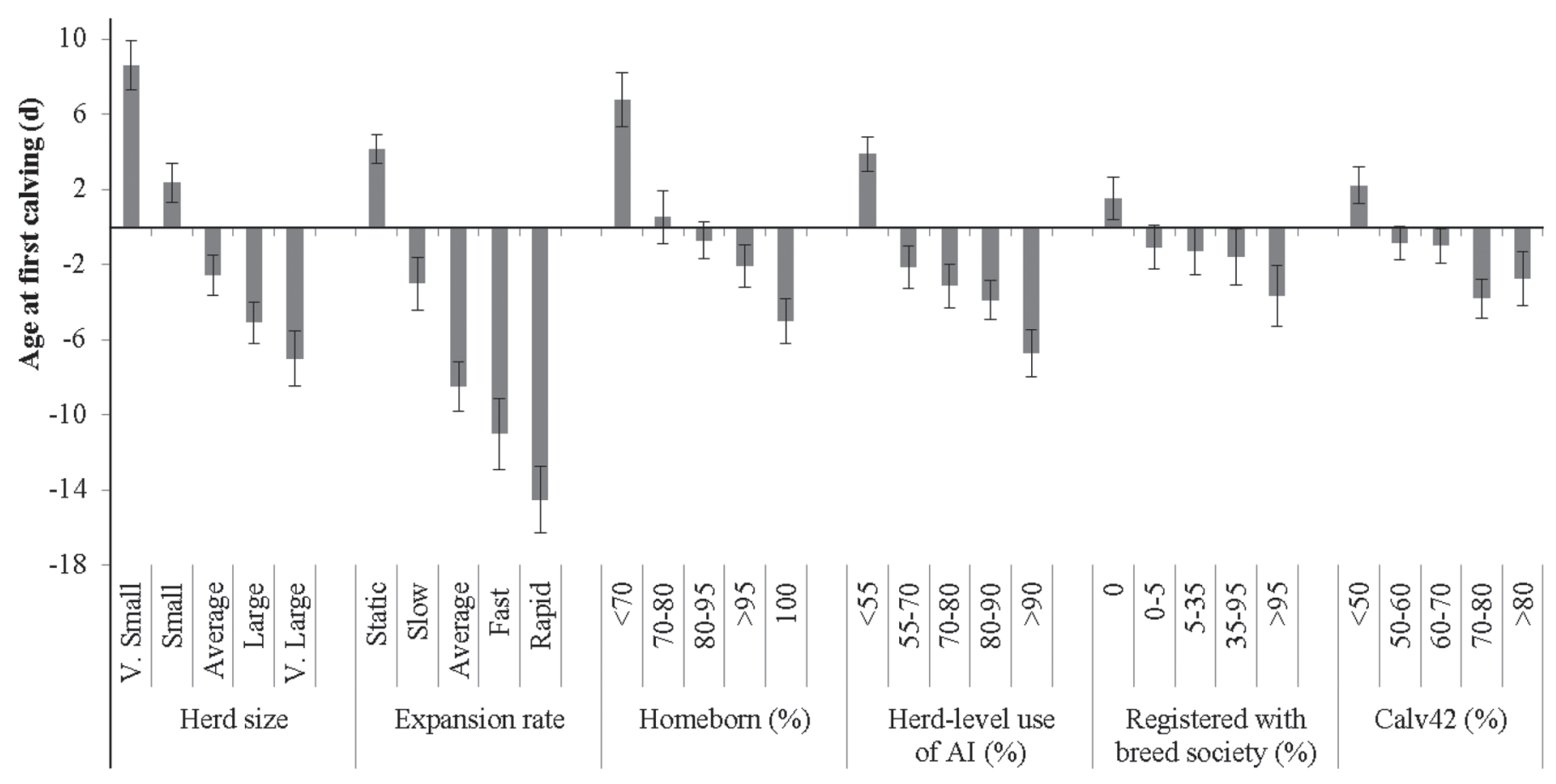

Figure 2. Mean (1 SE each side represented as error bars) best linear unbiased estimate for age at first calving for each category of 6 herdlevel characteristics. V. = very; Calv42 = the proportion of cows in the herd that calved in the first $42 \mathrm{~d}$ of the calving season. 


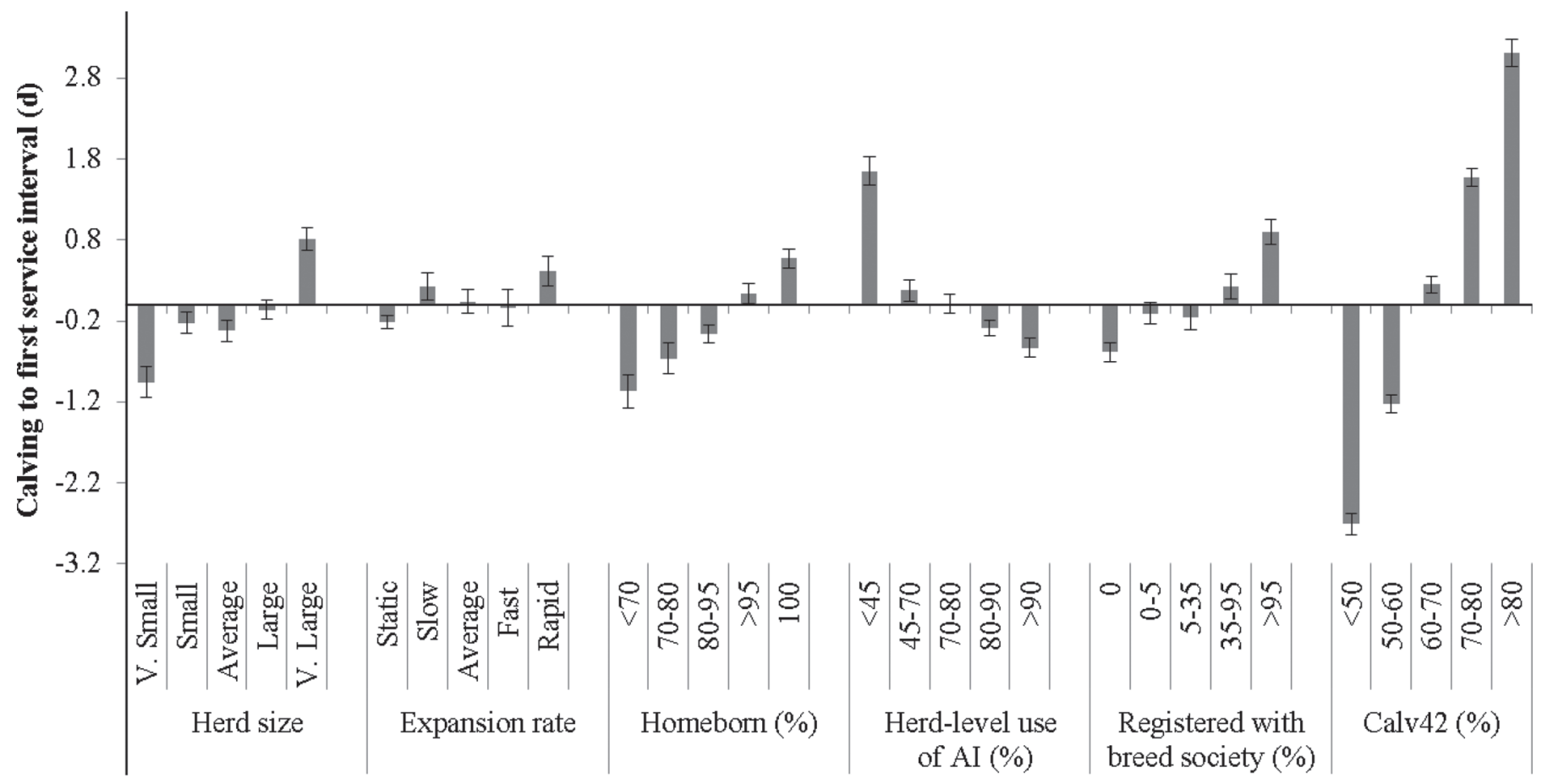

Figure 3. Mean (1 SE each side represented as error bars) best linear unbiased estimate for calving to first service interval for each category of 6 herd-level characteristics. V. = very; Calv $42=$ the proportion of cows in the herd that calved in the first $42 \mathrm{~d}$ of the calving season.

notypic performance independent of genetic merit. The overall goal was to establish the usefulness of BLUE in herd management decision support tools and what factors would need to be considered when attempting to explain differences in herd solutions to producers. For example, what was quite clear from the present study was that the mean herd performance for a range of traits differed substantially across geographical lo-

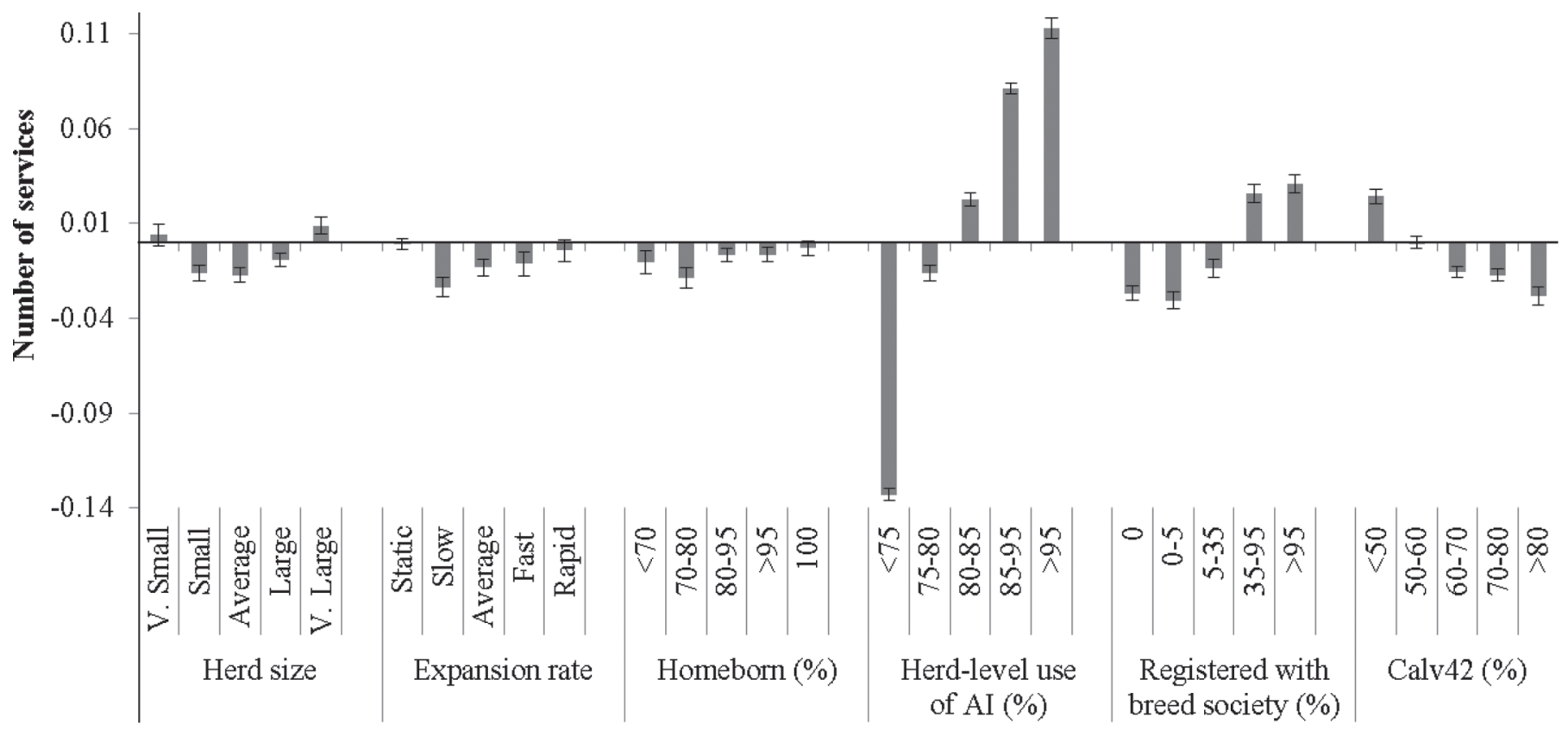

Figure 4. Mean (1 SE each side represented as error bars) best linear unbiased estimate for number of services for each category of 6 herdlevel characteristics. V. = very; Calv 42 = the proportion of cows in the herd that calved in the first $42 \mathrm{~d}$ of the calving season. 


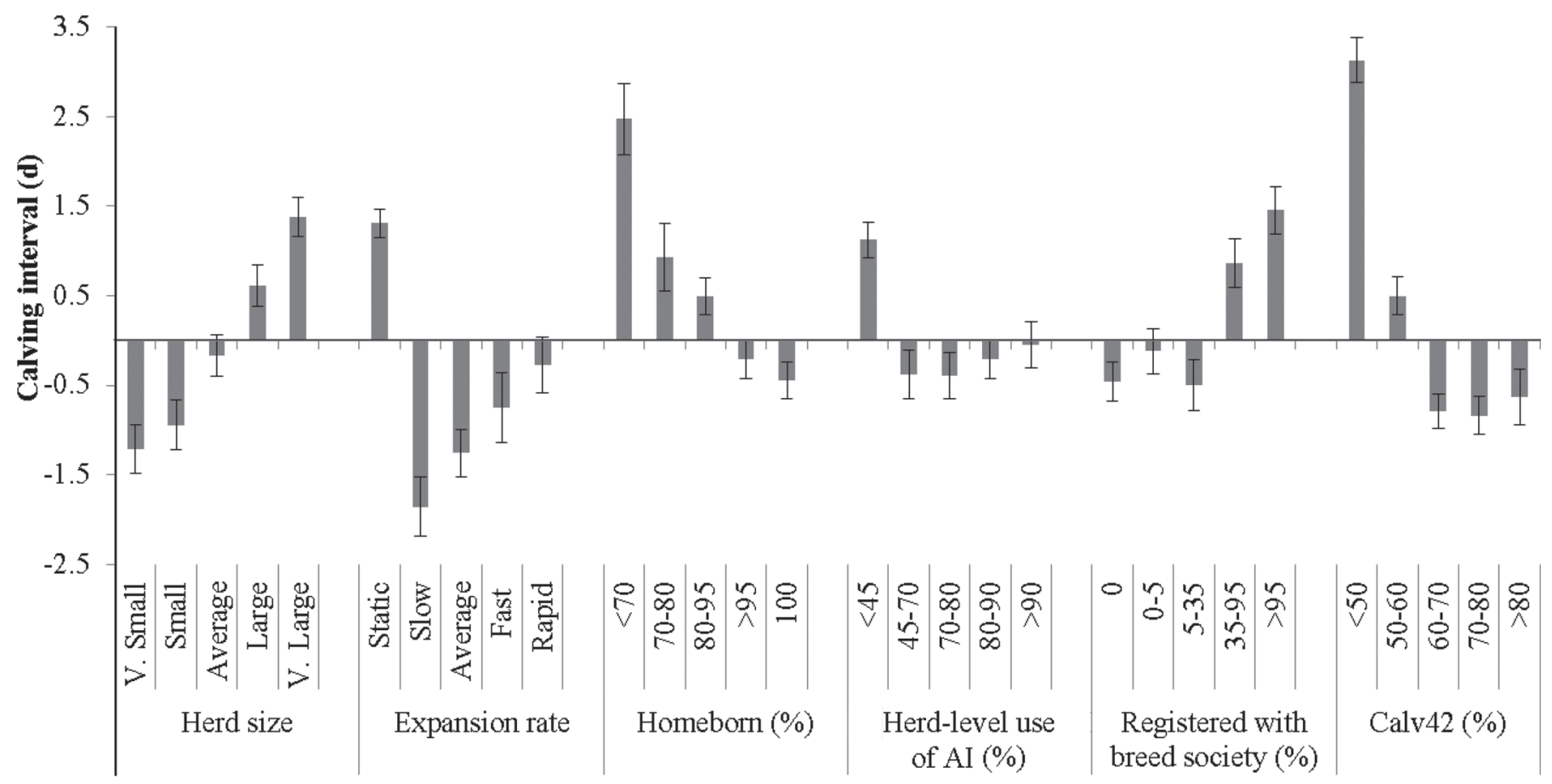

Figure 5. Mean (1 SE each side represented as error bars) best linear unbiased estimate for calving interval for each category of 6 herd-level characteristics. V. = very; Calv $42=$ the proportion of cows in the herd that calved in the first $42 \mathrm{~d}$ of the calving season.

cations, which could, of course, be attributable to a benchmarking herds on performance, first, differences multitude of factors, not least the climatic conditions in the mean genetic merit of the herd should be acpertinent to each geographical location. Hence, when counted for, and then consideration should be taken of

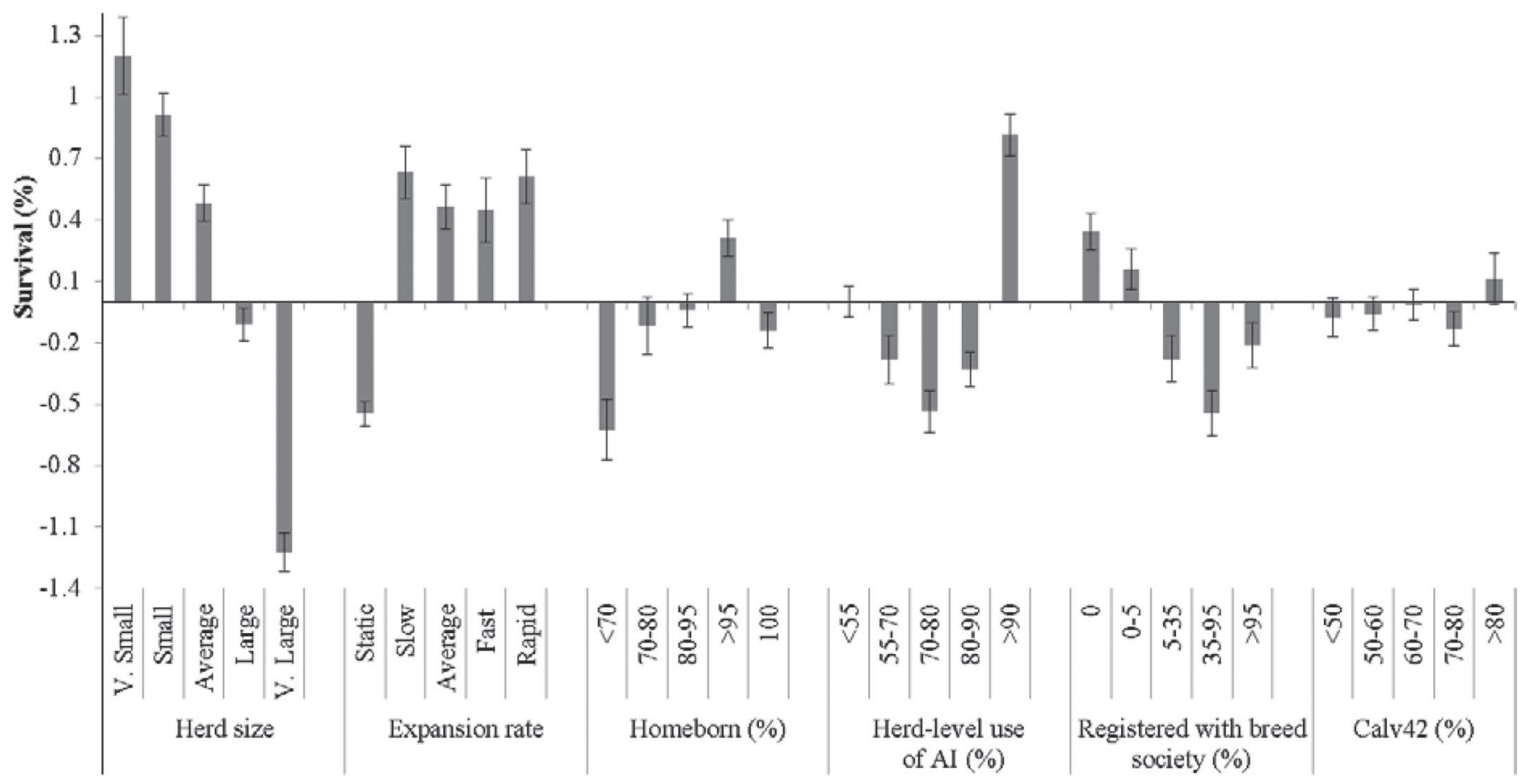

Figure 6. Mean (1 SE each side represented as error bars) best linear unbiased estimate for survival for each category of 6 herd-level characteristics. V. = very; Calv 42 = the proportion of cows in the herd that calved in the first $42 \mathrm{~d}$ of the calving season. 


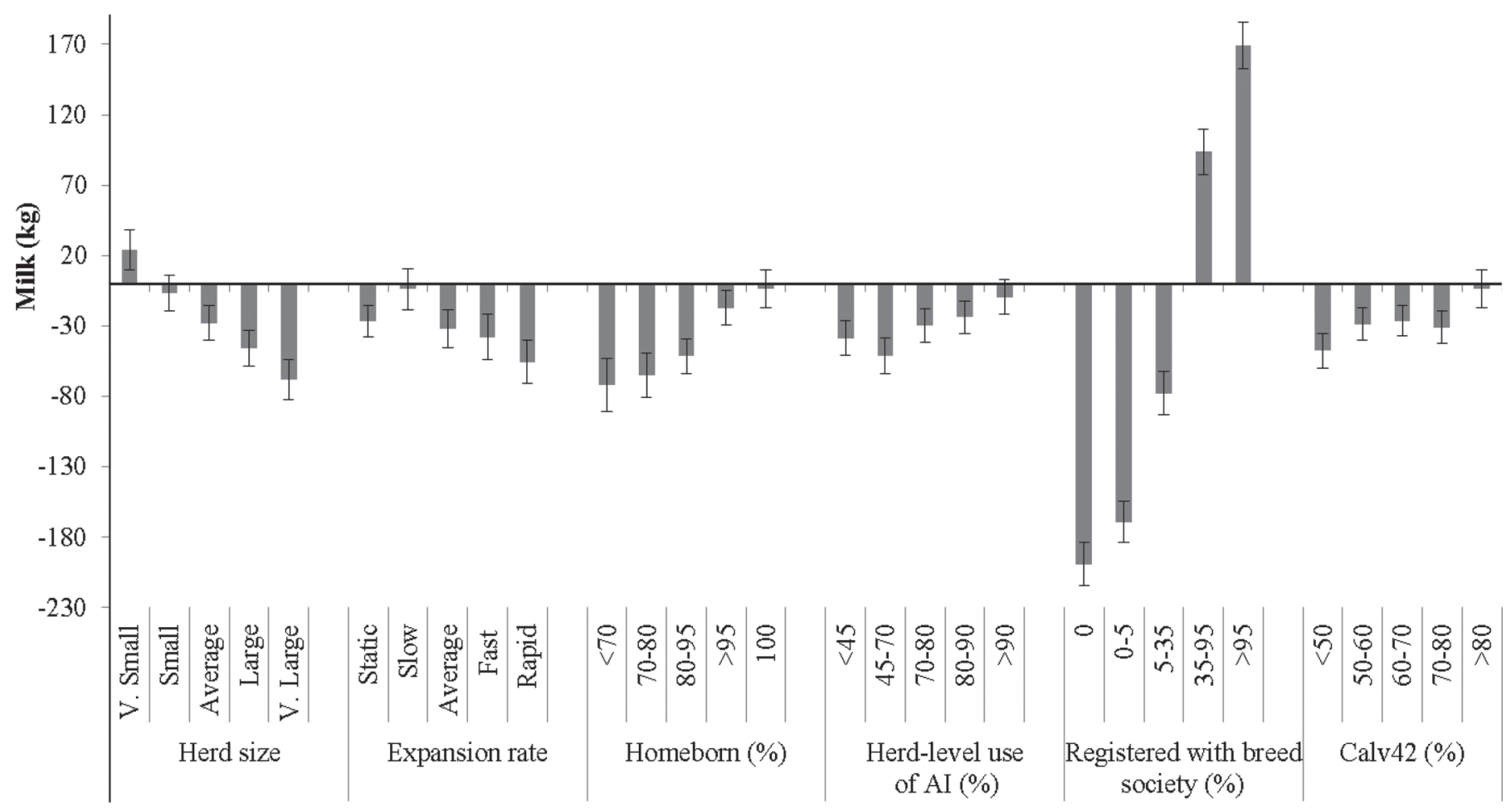

Figure 7. Mean (1 SE each side represented as error bars) best linear unbiased estimate for milk yield for each category of 6 herd-level characteristic. V. = very; Calv 42 = the proportion of cows in the herd that calved in the first $42 \mathrm{~d}$ of the calving season.

the geographical location as well as the other factors reported in the present study to be associated with herdyear BLUE solutions. Moreover, the expected effect on performance of a herd transitioning between different states of the herd-level factors (e.g., a rapidly expanding herd slowing down to eventually become a static herd) can be predicted and relayed to the producer as well as being used to explain why a herd's performance may differ from expectation.

\section{Mean Annual Best Linear Unbiased Estimates Versus Best Linear Unbiased Predictors}

Although the annual least squares means herd-year BLUE for calving interval tended to fluctuate by year, a linear regression line fitted through all $9 \mathrm{yr}$ BLUE revealed a mean annual reduction of $1 \mathrm{~d}\left(\mathrm{SE}=0.30, \mathrm{R}^{2}\right.$ $=0.63$ ). The linear regression coefficient fitted through all years of BLUP for calving interval was -0.33 (SE $\left.=0.01, \mathrm{R}^{2}=0.99\right)$, whereas the regression coefficient fitted to the raw annual calving interval values was $-1.54\left(\mathrm{SE}=0.29, \mathrm{R}^{2}=0.81\right)$. This indicates that the observed additive genetic improvement contributed to $21.75 \%$ of the improvement in the raw phenotype for calving interval, with BLUE contributing to a further $67.89 \%$. Similarly, the annual least squares means herdyear BLUE for milk yield also fluctuated by year; after a linear regression was fitted through the $10 \mathrm{yr}$ of data for milk yield, it revealed that from the sum of the BLUE and BLUP regression coefficients, BLUE contributed to $69 \%$ (regression coefficient $=17.69, \mathrm{SE}=$ $17.99, \mathrm{R}^{2}=0.11$ ) of the improvement in milk yield. Although it has previously been suggested that between the years 1980 and 2004, additive genetic merit was the main contributor $(63 \%)$ to the deterioration in calving interval of Irish dairy cow (Berry et al., 2014), the present study suggested the change in genetic merit is only contributing $21.75 \%$ to the improvement. Although the current relative contribution of additive genetic merit to the raw phenotype may be considered low in comparison to what was previously described, breeding programs still offer an opportunity for cumulative and permanent gains to be achieved with the performance of the current generation being a function of the genetic improvement in previous generations. Moreover, it should be noted that the approximate replacement rate on dairy farms is $20 \%$; therefore, only $20 \%$ of the herd being replaced annually will benefit from the change in genetic merit.

The repeatability of herd-year BLUE may be of particular interest for, not only the individual producer, but also the dairy processors and the wider dairy sector as a whole, owing to the predictability of the herd performance. The high repeatability of herd-year BLUE 
for milk yield implies that the herd performance of future years can be predicted with reasonable accuracy despite the likely contributions of external factors such as weather conditions, milk price volatility, and feed input costs. In the present study, $74 \%$ of the top $20 \%$ yielding dairy herds are predicted to remain in this category in the subsequent year (Table 3). The benefit of forecasting production would be to enable milk processors to predict the quantities of milk in the following production year, leading to more secure production contracts and manufacturing planning. The fact that the fertility traits in the present study (i.e., calving interval, calving to first service interval, and number of services) were lowly repeatable over years implies that extension service providers must continually emphasize the importance of consistently achieving key performance indicators as well-performing herds in any year may not necessarily perform well in subsequent years.

\section{Tailored Decision Support}

Extension services and the associated advice provided to producers on how to achieve key performance indicators have traditionally been relatively generic. In general, such advice has been to use the best germplasm available and adopt the associated best management practices. Anecdotally, in fact, poor breeding choices are often blamed for poor phenotypic herd performance, despite the choice of herd management practices in place on farm having a critical role in the realization of the genetic potential of the animals. There is clearly a gap in the market for tailored decision support tools to more distinctly differentiate between the contributing factors to the observed performance and thus where the resources should be exerted to achieve gains in performance. Bastin et al. (2009) recognized this necessity and proposed that milk urea concentrations in dairy cow milk could potentially be used as a tool to provide producers with feed management decision support owing to the relationship between milk urea and protein metabolism. Similarly, Caccamo et al. (2008) proposed that by identifying sources of herd-level variation in milk components and SCC, management decision support tools could be developed that would quantify how different levels of management contribute to the identified sources of variation. In fact, the benefit of using BLUE to disentangle the contribution of both genetic merit and management to the observed performance has the potential to be applicable to a whole range of different performance characteristics and species; Englishby et al. (2017), for example, proposed using BLUE from random regression models on carcass weight as a tool to quantify herd-level effects on carcass growth rates and other beef carcass trait profiles.
The usefulness and applicability of BLUE in the context of the data explored in the present study is illustrated, by means of an example, in Figure 8 for calving interval, just for the 2015 calendar year. Despite the herd-year raw mean phenotypic calving interval for 2 herds only differing by $0.42 \mathrm{~d}$ (i.e., herds 1 and 6 in Figure 8), clear differences in the BLUE for these herds existed after adjusting for the fixed and random effects in the genetic evaluation model; the fixed effects in the national genetic evaluations are age at calving, heterosis, and recombination loss. Based on the herds in Figure 8, the BLUE calving interval for herd 1 lengthened, whereas the BLUE calving interval for herd 6 shortened; the mean calving interval EBV of the cows in herds 1 and 6 in the year 2015, was $-5.57 \mathrm{~d}$ (very good) and $0.00 \mathrm{~d}$ (average), respectively. Therefore, the producers in herd 6 should focus more on breeding for superior fertility, whereas herd 1 may consider focusing on improving management to reap the benefit of its superior genetic merit; in this case, the farm advisor could attribute the suboptimal performance to some of the factors identified in the present study, for instance, expansion rate as well as other herd-level factors, such as level of heat detection monitoring. Using another example of 2 herds from Figure 8, herd 5 had the longest raw mean phenotypic calving interval $(13.23 \mathrm{~d})$, whereas herd 3 had the shortest raw mean phenotypic calving interval $(-4.42 \mathrm{~d})$; the respective BLUE for these herds ranked opposite $(+0.80$ and $+7.39 \mathrm{~d}$, respectively) after adjusting for the genetic merit of the herds (and other fixed effects in the genetic evaluation model).

When analyzing a sample set of herds in the year 2015 for milk yield, a similar phenomenon also existed when comparing raw herd means for milk yield and the respective BLUE (Figure 9). Unlike calving interval, the difference between the individual raw phenotypic performance and the corresponding herd BLUE was predominantly explained by the herds' EBV; this suggests that, depending on the trait, and therefore its heritability, the contribution of differences in EBV to the disparity between raw and BLUE differs. If the trait was highly heritable (e.g., milk yield), the herd's mean EBV explained the majority of the difference between the raw and BLUE; however, if the trait was lowly heritable (e.g., fertility; Berry et al., 2013), the difference between the raw and BLUE is only partially explained by herd mean EBV.

Two herd-level factors that were noticeably associated with herd BLUE for all traits investigated in the present study were expansion rate and herd size, both of which have also been previously reported to be associated with various performance traits in spring-calving dairy cow herds (Jago and Berry, 2011). Jago and Berry (2011) stated that faster expanding herds had a shorter 


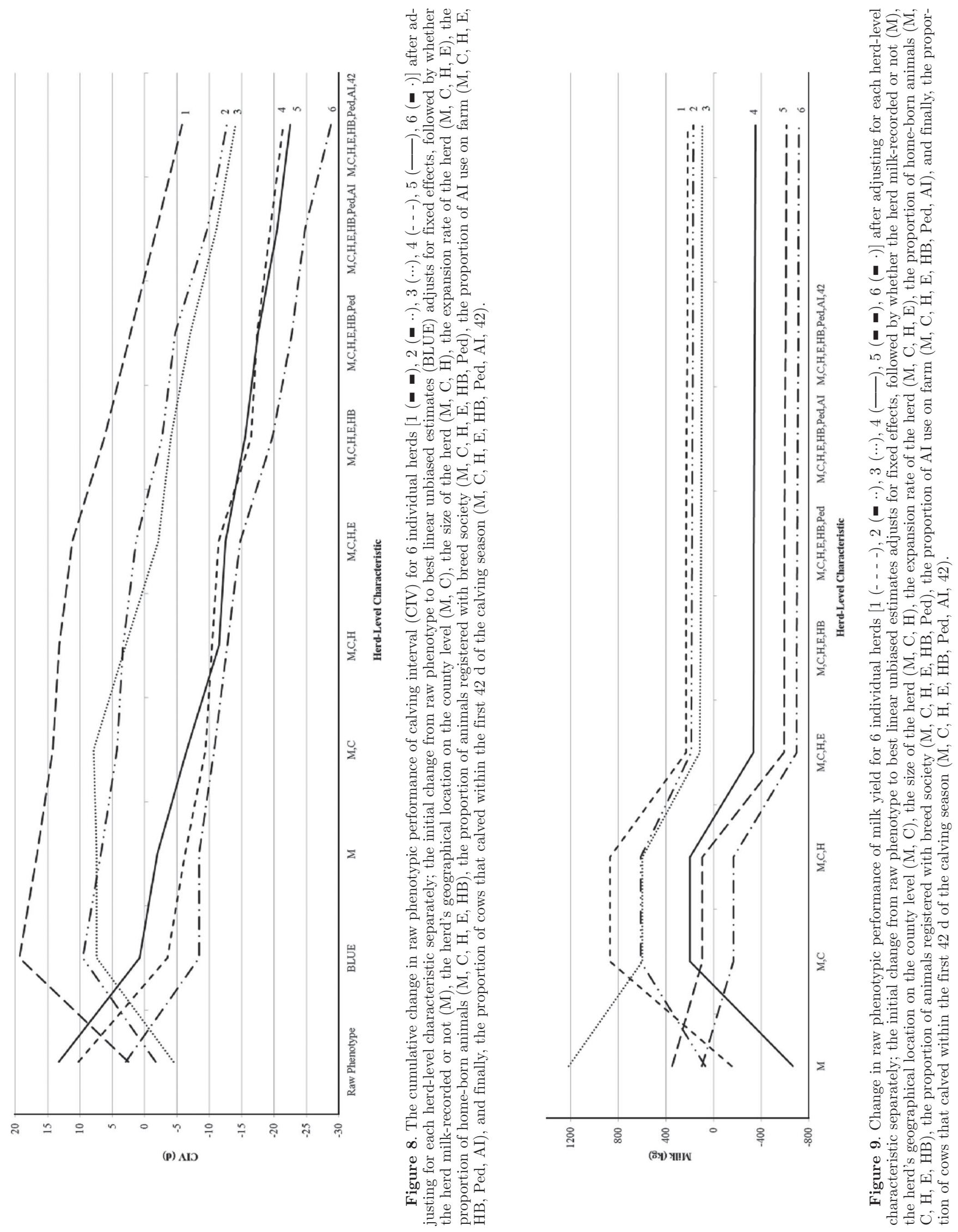


raw calving interval than static herds, and these findings were similar to the present study, which stated that slow, average, and fast rates of expansion were associated with shorter caving intervals in comparison to static herds. However, in direct contrast to Jago and Berry (2011), smaller herd sizes in the present study had, on average, shorter calving intervals.

The effect of geographical location on the mean BLUE for milk yield ranged from -365 to $1,915 \mathrm{~kg}$; the highest yielding county surrounded the country's capital and the majority of dairy producers in this location produce liquid milk achieved through the feeding of more concentrates; the lowest yielding region was predominantly a mountainous region. The effect of accounting for geographical location when explaining differences in BLUE was clearly evident when comparing the example herds in Figure 9 for milk yield. Changing farm location is not, of course, possible, but when producers are benchmarking key performance indicators, it is important that they are being compared with contemporaries, which in that case would imply altering the herd BLUE based on the model solutions for geographical location estimated in the present study. The remaining herd-level characteristics had minimal effects on the BLUE for milk yield.

\section{CONCLUSIONS}

Increased efficiency of production and optimizing the use of available resources should be based on pertinent advice tailored to each farm with a strategy on where to focus resources to achieve the greatest gains. At a macro-level, animal genetic merit and herd management are the 2 factors dictating the observed performance, and at the very least, a differentiation should be made between these as to which is the most likely limiting factor. At a management level, some factors (e.g., geographical location) are unavoidable and benchmarking of performance should take this into consideration; similarly, some factors may be transient such as herd expansion rate. The BLUE, and the appropriate analysis of such, can provide useful information to tailor decision support tools for individual herd managers. As BLUE are already routinely generated from (national) genetic evaluations, no extra resources are required other than to develop the decision support infrastructure. What is not yet known, however, is whether the extent of the association between the (estimated) genetic merit for a trait and its respective phenotypic performance differs depending on the herd
BLUE; such information would further the precision of breeding-specific support tools (i.e., matching the recommended germplasm to herd-specific conditions).

\section{ACKNOWLEDGMENTS}

Financial support from the Irish Department of Agriculture, Food and the Marine MultiRepro project is gratefully acknowledged.

\section{REFERENCES}

Bastin, C., L. Laloux, A. Gillon, F. Miglior, H. Soyeurt, H. Hammami, C. Bertozzi, and N. Gengler. 2009. Modeling milk urea of Walloon dairy cows in management perspectives. J. Dairy Sci. 92:3529-3540.

Berry, D. P., J. Kearney, K. Twomey, and R. Evans. 2013. Genetics of reproductive performance in seasonal calving dairy cattle production systems. Ir. J. Agric. Food Res. 52:1-16.

Berry, D. P., E. Wall, and J. Pryce. 2014. Genetics and genomics of reproductive performance in dairy and beef cattle. Animal 8(Suppl. 1):105-121.

Bozdogan, H. 2000. Akaike's information criterion and recent developments in information complexity. J. Math. Psychol. 44:62-91.

Caccamo, M., R. Petriglieri, G. Licitra, R. F. Veerkamp, M. H. Pool, and G. De Jong. 2008. Variance components for test-day milk, fat, and protein yield, and somatic cell score for analyzing management information. J. Dairy Sci. 91:3268-3276.

Calus, M. P. L., J. J. Windig, and R. F. Veerkamp. 2005. Associations among descriptors of herd managment and phenotypic and genetic levels of health and fertility. J. Dairy Sci. 88:2178-2189.

Crump, R., N. Wray, R. Thompson, and G. Simm. 1997. Assigning pedigree beef performance records to contemporary groups taking account of within-herd calving patterns. Anim. Sci. 65:193-198.

DAFM. 2016. AIM Bovine Statistics Report. Page 65. AIM Division, Department of Agriculture, Food and the Marine, Dublin, Ireland.

Englishby, T. M., K. L. Moore, D. P. Berry, M. P. Coffey, and G. Banos. 2017. Herd-specific random regression carcass profiles for beef cattle after adjustment for animal genetic merit. Meat Sci. 129:188-196.

Jago, J., and D. P. Berry. 2011. Associations between herd size, rate of expansion and production, breeding policy and reproduction in spring-calving dairy herds. Animal 5:1626-1633.

Meuwissen, T. H., B. Hayes, and M. Goddard. 2001. Prediction of total genetic value using genome-wide dense marker maps. Genetics 157:1819-1829.

MiX99 Development Team. 2015. MiX99: A software package for solving large mixed model equations. Vol. 2017. Biometrical Genetics, Natural Resources Institute Finland (Luke), Jokioinen, Finland.

Muir, W. 2007. Comparison of genomic and traditional BLUP-estimated breeding value accuracy and selection response under alternative trait and genomic parameters. J. Anim. Breed. Genet. 124:342-355.

Patry, C., and V. Ducrocq. 2011. Accounting for genomic pre-selection in national BLUP evaluations in dairy cattle. Genet. Sel. Evol. 43:30.

Schmitz, F., R. W. Everett, and R. L. Quaas. 1991. Herd-year-season clustering. J. Dairy Sci. 74:629-636.

van Bebber, J., N. Reinsch, W. Junge, and E. Kalm. 1997. Accounting for herd, year and season effects in genetic evaluations of dairy cattle: A review. Livest. Prod. Sci. 51:191-203. 